\title{
General analysis of type I second-harmonic generation with elliptical Gaussian beams
}

\author{
Tim Freegarde* and Julian Coutts ${ }^{\dagger}$ \\ Clarendon Laboratory, Parks Road, Oxford OX1 3PU, UK
}

Jochen Walz, Dietrich Leibfried, ${ }^{\dagger}$ and T. W. Hänsch

Max-Planck-Institut für Quantenoptik, Hans-Kopfermann-Strasse 1, 85748 Garching, Germany

Received December 2, 1996; revised manuscript received March 14, 1997

\begin{abstract}
One can enhance second-harmonic generation in angle-tuned crystals by focusing the fundamental to an elliptical waist in the crystal. We present a general analysis for type I phase-matched uniaxial and biaxial crystals that will find particular application in the generation of harmonics from diode lasers. (C) 1997 Optical Society of America [S0740-3224(97)01308-8]
\end{abstract}

\section{INTRODUCTION}

The optimal focusing arrangement for second-harmonic generation is a compromise between the focal intensity and the depth of focus (confocal parameter) within the crystal. In the absence of birefringence (noncritical phasematching), the fundamental beam should have a circular cross section and a confocal parameter of 0.35 of the crystal's length. ${ }^{1}$

Angle-tuned (critical) phase matching causes the cylindrical symmetry of harmonic generation to be lost, and elliptical (or anamorphic) focusing into the crystal has been shown to be advantageous. In this paper we present a theoretical analysis of type I phase matching and conclude that the harmonic power can be enhanced by as much as $30 \%$. The less common type II phase matching, in which the fundamental is split between ordinary and extraordinary polarizations, requires a rather different analysis if these components are collinear ${ }^{2}$; a proposed noncollinear scheme is, however, equivalent to the type I arrangement considered here. ${ }^{3}$

Elliptical focusing will be especially useful for the frequency doubling of diode lasers, ${ }^{4}$ for not only is the beam profile usually elliptical but any enhancement cavities are likely to be astigmatic. To match such a geometry efficiently, or at least to be allowed a degree of insensitivity, will be useful indeed. Such considerations will be yet more important when frequency-doubling or -mixing stages are cascaded, as with frequency tripling ${ }^{5}$ and quadrupling. 6,7 Our results will also be of particular relevance to second-harmonic generation by use of Brewsterangled crystals that render a circular incident beam elliptical. For high-power lasers as well, if the optimal circular focus leads to crystal damage ${ }^{8}$ or to thermally induced dephasing, elliptical focusing may provide a solution.

Cylindrical focusing was first considered for secondharmonic generation by Volosov and Nilov, ${ }^{9}$ Volosov, ${ }^{10}$ and Chmela, ${ }^{11}$ but true elliptical arrangements were not addressed until Librecht and Simons ${ }^{12}$ considered a specific example and analyzed the asymptotic behavior. Elliptical geometries had in the meantime been applied to parametric oscillation by Kuizenga, ${ }^{13}$ and further analyses followed. ${ }^{14,15}$ Pure cylindrical focusing has been used with pulsed Nd:YAG lasers ${ }^{16}$ and with copper-vapor lasers, ${ }^{17}$ for which a limited theoretical analysis has been presented $^{18}$ and proposed for the reduction of thermal dephasing. ${ }^{19}$ Interest in elliptical focusing has reawoken with the advent of the diode laser, first with the investigation of a specific example of type II phase matching ${ }^{20}$ and then with the qualitative matching of an elliptical beam into an enhancement cavity ${ }^{21}$; the latter technique was also partly addressed in conjunction with titaniumdoped sapphire lasers. ${ }^{22,23}$ Recently Steinbach et al. ${ }^{24}$ showed excellent agreement between modeled and experimental doubling of a cw argon-ion laser and calculated some more general results.

This is clearly, then, not the first attempt to treat elliptical focusing in second-harmonic generation. Whereas previous analyses generally referred to specific experimental situations, however, the treatment presented here leads to results of general significance as a function of the usual crystal parameter $B$, from which the optimal elliptically focused geometries can readily be found and for which generally applicable recipes are presented. Often, of course, it may not be experimentally practicable to use the ideal arrangement, and the conversion efficiency indeed appears to be a gentle function of the focusing parameters. We therefore present curves for nonoptimal ellipticities from which the best focusing strengths and expected efficiencies can be derived.

Finally, in various limiting regimes, some physical interpretation of the results is possible. For many nonlinear crystals the birefringence is high enough that an analytic calculation for the limit of high birefringence is a 
good approximation. The treatment given here, which reduces the calculation to tabulated functions, follows that of Librecht and Simons, ${ }^{12}$ although it is appropriate not only in the extremes of strong and weak focusing but also in the interesting regime where the conversion efficiency is maximum.

\section{THEORY}

Second-harmonic generation is most efficient when it is phase matched; that is, the material dispersion between the fundamental and the harmonic wavelengths is compensated such that, when the harmonic contributions from all points within the crystal are added coherently into the output beam, the maximum intensity is achieved. For plane waves this requires that the components all emerge in phase and thus that the refractive indices for the fundamental and the harmonic be equal. For focused beams there should be a small mismatch to correct for the Guoy shift, ${ }^{25}$ which can be considered to conserve the momentum of the light beam as it passes through a focus.

Type I phase matching exploits the birefringence of the nonlinear crystal, which is oriented such that the refractive index for one polarization at the fundamental wavelength matches that at the harmonic in the other. If the refractive indices for the two polarizations are represented as functions of the angular coordinates by surfaces, then phase matching occurs when the ellipsoids thus formed meet. If these surfaces intersect, the line of intersection corresponds to the locus of phase-matched crystal orientations, and rotation normal to this line allows the crystal to be angle tuned, the orientation chosen being that point on the locus that provides the largest nonlinear coefficient. Except in the case of noncritical, or $90^{\circ}$, phase matching-which involves bringing the ellipsoids to a single grazing contact by varying the crystal temperature - the phase match will be highly sensitive to rotation away from the line of intersection of the ellipsoids, causing a narrow angular acceptance for the incident light, but will be insensitive to rotation along this line.

The induced field at the harmonic frequency depends quadratically on that of the fundamental, and the efficiency of second-harmonic generation thus increases as the input power is focused into a smaller area. If the constraints of phase matching can be neglected, the optimal focusing arrangement will be a compromise that optimizes both the electric-field strength at the focus and the longitudinal distance over which the focus extends and occurs when the confocal parameter of the Gaussian beam is 0.35 of the crystal length. With critical phase matching, however, only a central longitudinal slice of the beam falls within the narrow acceptance angle, and for optimal conversion the focusing must be weakened. The correspondingly larger focal region can be regarded as better able to accommodate Poynting-vector walk-off between the beams. The optimal parameters for a circular Gaussian beam in any such arrangement are described in a classic paper by Boyd and Kleinman. ${ }^{1}$

\section{ANALYSIS}

Our approach has been to generalize the circular beam analysis of Boyd and Kleinman, ${ }^{1}$ using instead an ellipti- cal, astigmatic form of the Gaussian beam to describe the fundamental and generated harmonics. We preserve the original notation of Boyd and Kleinman but have chosen to present our results in SI units. In extending the treatment to allow the Gaussian beams to have an elliptical cross section, we have added $x$ and $y$ subscripts where the elliptical asymmetry renders it necessary. The optic axis is in the $x-z$ plane.

We start, therefore, with an elliptical Gaussian beam, propagating with wave number $k_{1}=2 \pi \eta_{1} / \lambda$ along the $z^{\prime}$ axis, with an electric field given by

$$
E=E_{0} \frac{\exp \left(i k_{1} z^{\prime}\right) \exp \left[-\left(\frac{x^{\prime 2} / w_{x}{ }^{2}}{1+i \tau_{x}{ }^{\prime}}+\frac{y^{\prime 2} / w_{y}{ }^{2}}{1+i \tau_{y}{ }^{\prime}}\right)\right]}{\left[\left(1+i \tau_{x}{ }^{\prime}\right)\left(1+i \tau_{y}{ }^{\prime}\right)\right]^{1 / 2}},
$$

where $\tau_{x}{ }^{\prime}=2\left(z^{\prime}-f_{x}\right) / k_{1} w_{x}{ }^{2}$ and $\tau_{y}{ }^{\prime}=2\left(z^{\prime}-f_{y}\right) /$ $k_{1} w_{y}{ }^{2}$. The foci in the $y-z$ and $x-z$ planes are found at $z=f_{x}$ and $z=f_{y}$, respectively, thus permitting the beam to be astigmatic when the foci do not coincide, and the waist radii or ellipse semiaxes are $w_{x}$ and $w_{y}$, respectively.

Birefringence in the nonlinear crystal will be characterized by the walk-off angle $\rho$ between the fundamental and the harmonic. For type I phase matching in negative uniaxial crystals, where the ordinary (o) and the extraordinary $(e)$ refractive indices at the fundamental and the harmonic frequencies are given by $\eta_{o}^{\omega}, \eta_{e}^{\omega}, \eta_{o}^{2 \omega}$ and $\eta_{e}^{2 \omega}$, the walk-off angle is given by

$$
\tan \rho=\frac{1-\left(\eta_{o}^{2 \omega} / \eta_{e}^{2 \omega}\right)^{2}}{\cot \theta+\tan \theta\left(\eta_{o}^{2 \omega} / \eta_{e}^{2 \omega}\right)^{2}},
$$

where the phase-matching angle $\theta$ satisfies

$$
\sin ^{2} \theta=\frac{\left(\eta_{o}^{\omega}\right)^{-2}-\left(\eta_{o}^{2 \omega}\right)^{-2}}{\left(\eta_{e}^{2 \omega}\right)^{-2}-\left(\eta_{o}^{2 \omega}\right)^{-2}} .
$$

Similar expressions apply for positive uniaxial crystals, with the $\omega$ and $2 \omega$ labels interchanged, and the treatment presented here is valid for both. With biaxial crystals $^{26,27}$ there are rather too many configurations to describe here, but one can derive a value for the walk-off angle $\rho$ by using

$$
\begin{aligned}
\tan \rho & =\frac{1}{k_{2}} \frac{\mathrm{d}(\Delta k)}{\mathrm{d} \phi} \\
& =\frac{1}{\eta} \frac{\mathrm{d}}{\mathrm{d} \phi}\left[\eta^{\omega}(\phi)-\eta^{2 \omega}(\phi)\right],
\end{aligned}
$$

where $\phi$ is the angular coordinate in the critical direction, $\eta^{\omega}(\phi)$ and $\eta^{2 \omega}(\phi)$ are the angle-dependent refractive indices at the fundamental and the harmonic frequencies, respectively, and $\Delta k$ is the wave-number mismatch, $2 k_{1}-k_{2}$.

We now apply the analysis of Boyd and Kleinman, using the more general elliptical form of the Gaussian beam. The fundamental electric field at a given distance into the crystal is given by Eq. (1) together with a term to allow for absorption, and the resulting polarization at the harmonic frequency is found to have a transverse elliptical 
Gaussian dependence. Each slice will thus propagate as an elliptical Gaussian beam, and all such beams can be added coherently further downstream; walk-off is included by making a shear coordinate transformation. Our version of the variables used in this treatment is summarized as follows:

$$
\begin{aligned}
& \omega_{1}, \omega_{2}, \quad \text { fundamental and harmonic frequencies; } \\
& k_{1}, k_{2} \text {, fundamental and harmonic wave numbers; } \\
& \Delta k, \quad \text { wave-number mismatch, } 2 k_{1}-k_{2} \text {; } \\
& w_{x}, w_{y} \text {, waist radii (ellipse semiaxes); } \\
& b_{x}, b_{y}, \quad \text { confocal parameters, } w_{x}{ }^{2} k_{1}, w_{y}{ }^{2} k_{1} \text {; } \\
& l \text {, crystal length; } \\
& \xi_{x}, \xi_{y}, \quad \text { focusing parameters, } l / b_{x}, l / b_{y} \text {; } \\
& \text { e, } \quad \text { ellipticity, } w_{x} / w_{y} \text {; } \\
& \mu \text {, focus position in the critical }(x) \text { direction } \\
& =\left(l-2 f_{x}\right) / l \text { when the focus is at } z=f_{x} ; \\
& \Delta, \quad \text { astigmatic distance between beam waists } \\
& =2\left(f_{x}-f_{y}\right) /\left(k_{1} w_{y}^{2}\right) \text {; } \\
& \rho, \quad \text { birefringent walk-off angle in the crystal; } \\
& \beta, \quad \text { ratio of walk-off angle to Gaussian-beam } \\
& \text { half-angle, }=\rho w_{x} k_{1} / 2 \text {. }
\end{aligned}
$$

$\mathbf{e}$ is thus the ellipticity of the Gaussian beam waist. Two dimensionless coordinate systems are used: $s$ and $s^{\prime}$ are angles from the axis in the critical and noncritical planes, in units of Gaussian half-angle; $\tau_{x}, \tau_{x}{ }^{\prime}, \tau_{y}$, and $\tau_{y}{ }^{\prime}$ are longitudinal dimensions in units of the confocal parameter. The remaining variables are essentially unchanged:

$$
\begin{array}{ll}
\sigma_{x}, & \text { phase mismatch in the critical direction } \\
& =1 / 2 b_{x} \Delta k ; \\
& =\sigma_{x}+4 \beta s ; \\
\sigma^{\prime} & =2 \omega_{1}^{2} d_{\text {eff }}^{2} / \pi \epsilon_{0} c^{3} \eta_{1}^{2} \eta_{2} ; \\
K & \text { fundamental power; } \\
P_{1}, & \text { absorption coefficient for fundamental; } \\
\alpha_{1}, & \text { absorption coefficient for harmonic; } \\
\alpha_{2}, & =\alpha_{1}-1 / 2 \alpha_{2} ; \\
\alpha & =\alpha_{1}+1 / 2 \alpha_{2} ; \\
\alpha^{\prime} & =1 / 2 \alpha b_{x} . \\
\kappa &
\end{array}
$$

The relations among the on-axis focal harmonic polarization $P_{0 x}$, the fundamental electric field $E_{0}$, and the total fundamental power $P_{1}$ are given by

$$
\begin{aligned}
P_{0 x} & =\epsilon_{0} d_{\mathrm{eff}} E_{0}^{2} \\
& =4 P_{1} d_{\mathrm{eff}} /\left(\pi \eta_{1} c w_{x} w_{y}\right),
\end{aligned}
$$

and the second harmonic intensity $S$ is given in terms of the field strength $E_{2}$ by

$$
S=1 / 2 \quad \epsilon_{0} \eta_{2} c\left|E_{2}\right|^{2} .
$$

We ultimately reach an expression for the harmonic power:

$$
P_{2}=K P_{1}^{2} l k_{1} \exp \left(-\alpha^{\prime} l\right) h_{m},
$$

where all the optimizable parameters are contained within the term

$$
h_{m}=\frac{\pi^{2}}{\xi_{x}} \exp (\mu \alpha l)\left[\frac{2}{\pi^{1 / 2}} \mathbf{e} \int_{-\infty}^{\infty} \exp \left(-4 s^{2}\right)|H|^{2} \mathrm{~d} s\right],
$$

where

$$
H=\frac{1}{2 \pi} \int_{-\xi_{x}(1-\mu)}^{\xi_{x}(1+\mu)} \frac{\exp \left(-\kappa \tau_{x}{ }^{\prime}\right) \exp \left(i \sigma^{\prime} \tau_{x}{ }^{\prime}\right)}{\left(1+i \tau_{x}{ }^{\prime}\right)^{1 / 2}\left[1+i\left(e^{2} \tau_{x}{ }^{\prime}+\Delta\right)\right]^{1 / 2}} \mathrm{~d} \tau_{x}{ }^{\prime}
$$

Although we have chosen throughout to refer primarily to properties in the critical $x$ direction, a similar pair of expressions results if we work instead with properties in the $y$ direction and make appropriate changes to our definitions of $\beta, \kappa, \mu, \sigma^{\prime}$, and $\mathbf{e}$.

$h_{m}$ is a function of the parameters $\xi_{x}, \mathbf{e}, \beta, \sigma_{x}, \mu, \Delta, l$, and $\alpha$. The last term characterizes the absorption of the crystal and may often be assumed to be zero, in which case the optimal values of $\mu$ and $\Delta$ are found to be zero and the dependence of $h_{m}$ on $l$ will disappear. $\sigma_{x}$ represents the deviation from normal phase matching and will in practice be optimized by fine adjustment of the crystal orientation, so only the optimal value of $\sigma_{x}$ need be considered. The set of parameters necessary for determining $h_{m}$ is thus reduced to $\xi_{x}, \mathbf{e}$, and $\beta$, and it is convenient to rewrite $\beta$ in the form

$$
\beta=B\left(\xi_{x}\right)^{-1 / 2}
$$

where $B$ is completely defined by the physical properties of a given crystal through $B=1 / 2 \rho\left(l k_{1}\right)^{1 / 2}$. $B$ is thus the ratio of the walk-off angle to the 1/e amplitude halfangle of a Gaussian beam whose confocal parameter equals the crystal length; alternatively, $B$ is $\sqrt{1.39}$ times this ratio for a beam that would be the optimum under noncritical phase matching. The function $h_{m}$ is thus defined by $B, \xi_{x}$, and $\mathbf{e}$, where the first parameter describes the crystal and the others describe the focusing arrangement, which is under our control.

Equation (8) correctly reduces to the result of Boyd and Kleinman for circular focusing and is consistent with those of Steinbach et al. ${ }^{24}$ when there is no absorption and of Librecht and Simons ${ }^{12}$ when in addition the focus is at the crystal center. The formula of Bourzeix et al. ${ }^{23}$ describes the behavior in the limit $\mathbf{e} \rightarrow 0$ and provides a reasonable estimate of the efficiency for $\mathbf{e}<1$ but is inappropriate at the optimal ellipticity and above and cannot be used to derive the optimal conditions.

For the numerical calculation we first expand Eqs. (9) and (10) as a triple integral over $s$ and $\tau_{x}{ }^{\prime}$ (twice, as $\tau$ and $\left.\tau^{\prime}\right)$, which allows the $s$ integral to be eliminated and renders the remaining double integral better behaved. By making the transformations $X=\tau-\tau^{\prime}$ and $Y=\tau+\tau^{\prime}$ $-2 \xi_{x} \mu$, we move the range in which the integrated function is large onto the $X=0$ axis. We then compute $h_{m}$ numerically, using the amoeba downhill simplex $\operatorname{method}^{28}$ to maximize the result for specific $B$ as a function of $\xi_{x}, \sigma_{x}$, and e. For the results presented, we have set the absorption to zero and placed the foci at the center of the crystal, which makes the calculation slightly easier and gives the results some general significance. 


\section{RESULTS AND INTERPRETATION}

The maximized values of $h_{m}$ for circular and optimal elliptical focusing are shown in Fig. 1 as functions of the crystal parameter $B$, and the corresponding focusing parameters, $\xi$ for circular and $\xi_{x}$ and $\xi_{y}$ for elliptical focusing, are shown in Fig. 2. The optimal ellipticity is indicated by the heavy solid curves of Figs. 3 and 4 , which show the values of $h_{m}$ and, respectively, $\xi_{y}$ and $\xi_{x}$ as functions of both $B$ and $\mathbf{e}$ and permit the performance of geometries with nonoptimal ellipticity to be found. The observed improvement in conversion efficiency, approaching a factor of $1.3,{ }^{21,24,29}$ is indeed predicted for elliptical focusing in crystals with appreciable birefringence and corresponds as expected to stronger focusing in the noncritical direction and to a broader focus in the critical direction. In such cases the conversion efficiency is little affected by a further increase in ellipticity, and it is found that $w_{y}$ remains fixed near its optimal value and thus that $\xi_{y}=\mathbf{e}^{2} \xi_{x}$ remains constant, as described by Steinbach et al. ${ }^{24}$; for suboptimal ellipticities, however, $\xi_{y}$ should decrease to meet the results of Boyd and Kleinman at $\mathbf{e}=1$.

Our results can be compared with those for parametric gain, ${ }^{13}$ which corresponds to generation of the harmonic in a particular Gaussian mode. Our cases of circular and elliptical focusing are given, respectively, by Kuizenga's configurations $^{13}$ for circular and elliptical probe beams with elliptical signal and idler, and $\alpha_{12 m}$ corresponds to

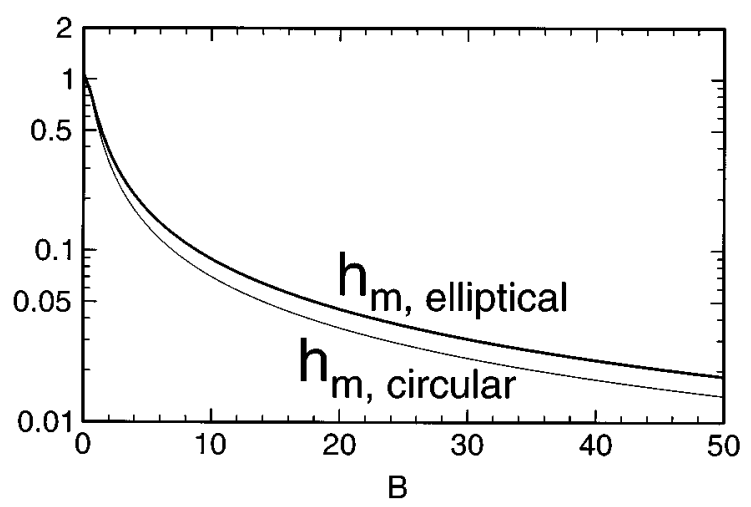

Fig. 1. Optimized conversion efficiency parameter $h_{m}$ for circular and elliptical Gaussian beams as a function of the crystal parameter $B$.

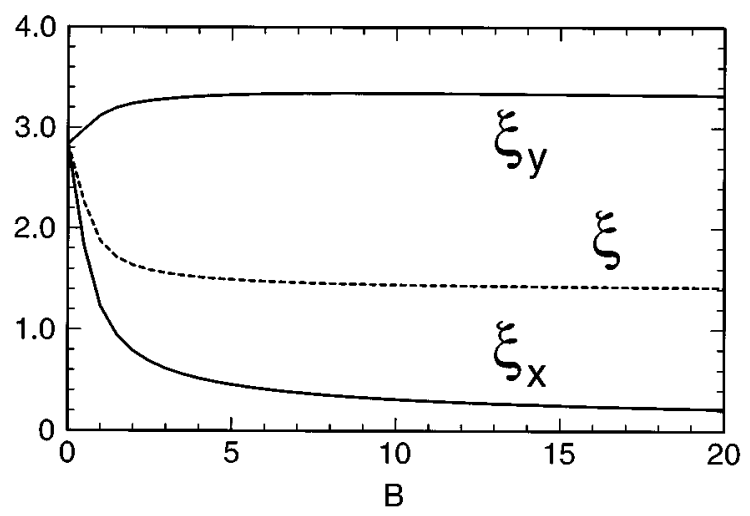

Fig. 2. Optimal focusing parameters $\xi_{x}$ and $\xi_{y}$ for elliptical Gaussian beams and $\xi$ for circular focusing.

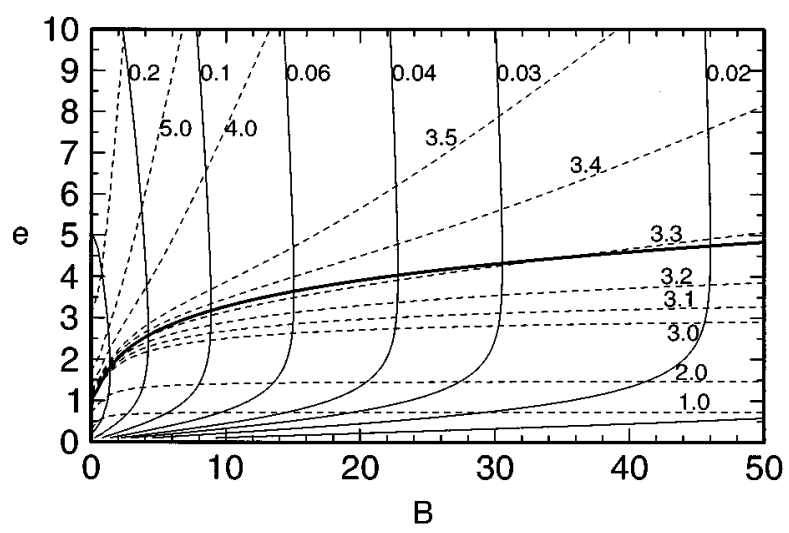

Fig. 3. Contours of $h_{m}$ (solid curves) and the focusing parameter $\xi_{y}$ (dashed curves) as functions of ellipticity e and crystal parameter $B$ for optimized second-harmonic generation with elliptical Gaussian beams. The heavy solid curve shows the optimal ellipticity as a function of $B$. Contour values are $0.02,0.03,0.04$, 0.06, 0.1, 0.2 and 0.5 for $h_{m}$ and 1.0, 2.0, 3.0, 3.1, 3.2, 3.3, 3.4, 3.5, 4.0, 5.0 and 10.0 for $\xi_{y}$.

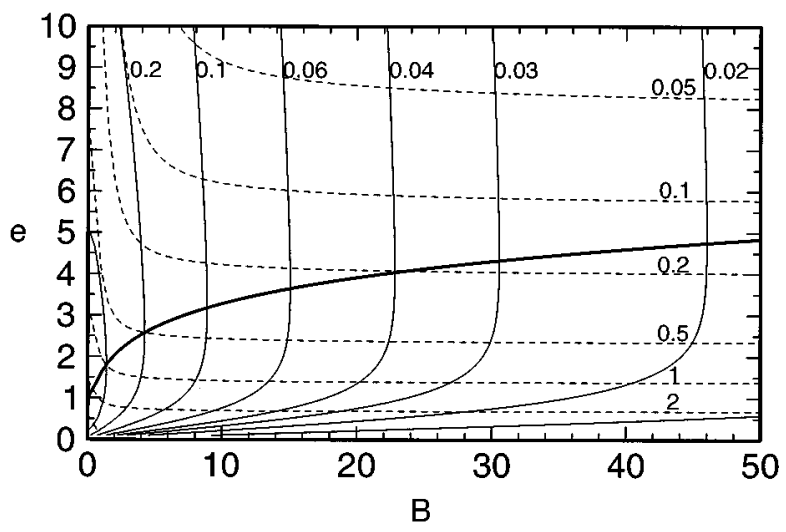

Fig. 4. Contours of $h_{m}$ (solid curves) and the focusing parameter $\xi_{x}$ (dashed curves) as functions of ellipticity e and crystal parameter $B$ for optimized second-harmonic generation with elliptical Gaussian beams. The heavy solid curve shows the optimal ellipticity as a function of $B$. Contour values are 0.02, 0.03, 0.04, 0.06, 0.1, 0.2 and 0.5 for $h_{m}$ and 0.05, 0.1, 0.2, 0.5, 1.0, 2.0 and 5.0 for $\xi_{x}$.

the reciprocal of our ellipticity e. Kuizenga's results, which closely follow ours, also indicate the ellipticity of the harmonic beam $1 /\left(\alpha_{12} \sqrt{\alpha_{x}}\right)$. At high $B$, the parametric gain calculations indicate an optimal efficiency approximately $10 \%$ lower than ours, consistent with an experimentally determined coupling efficiency of $87 \% .^{7}$

Boyd and Kleinman ${ }^{1}$ derive asymptotic forms for the dependence of $h_{m}$ on $\xi_{x}$ for the cases of weak and strong birefringence and focusing. Without birefringent walkoff (noncritical phase matching), weak focusing corresponds to the case of bounded plane waves when the harmonic power varies as the square of the crystal length and hence, for given $w_{x, y}$, in proportion to $\xi_{x}$; with strong focusing, the focal region lies completely within the crystal, and the independence of the harmonic power from the crystal length requires that $h_{m}$ vary in inverse proportion to $\xi_{x}$. With strong birefringence, walk-off limits the range of coherent addition to a length $l_{a}=\pi^{1 / 2} w_{x} / \rho$, and the asymptotic dependences thus become $\sqrt{\xi_{x}}$ and $1 / \sqrt{\xi_{x}}$. 
Such an analysis can be applied to elliptical Gaussian beams. For the case of high $B$ and $\mathbf{e} \gg 1$, we assume the harmonic intensity to correspond to the incoherent sum of coherent parcels of length $l_{a}$ within an overall length $b_{y}$ (the beam is more strongly focused in the noncritical $y$ direction), and we take the fundamental intensity to be constant in this region and confined to an area with the waist dimensions. The harmonic is assumed to fill an angular divergence matching that of the fundamental. Combining these terms gives a second-harmonic power of

$$
\begin{aligned}
P_{2} & \propto\left[\left(\frac{P_{1}}{w_{x} w_{y}}\right) w_{x} w_{y} l_{a}\left(\frac{b_{y}}{l_{a}}\right)^{1 / 2}\right]^{2}\left(\frac{1}{w_{x} w_{y}}\right), \\
& \propto P_{1}^{2} w_{y} / \rho .
\end{aligned}
$$

Effectively, we have decoupled the $x$ and $y$ dependences, so $w_{y}$ takes its optimal value and the efficiency is independent of $w_{x}$. Inasmuch as $P_{2} \propto l h_{m}$,

$$
h_{m} \propto 1 / B \sqrt{\xi_{y}} .
$$

This behavior is apparent in the upper-right-hand quadrant of Fig. 3, where $\xi_{y}$ is essentially constant and $h_{m}$ is independent of ellipticity. The analysis is, however, valid only when $l_{a} \ll b_{y}$, corresponding to the condition that $\mathbf{e} \ll B \sqrt{\xi_{y}}$, which accounts for the existence of an optimal ellipticity. Beyond this limit, the beam is cylindrically focused and the efficiency varies in inverse proportion to the ellipticity.

The derivation of relation (14) corresponds to the Boyd-Kleinman case of high $\beta$, whereby with increasing birefringence it becomes appropriate to consider only the part of the Gaussian whose direction lies within the acceptance angle of the crystal. In this regime, Boyd and Kleinman find that an analytic solution is possible; the same is true in the generalized case with elliptical Gaussian beams.

\section{ANALYTIC SOLUTION FOR HIGH $\beta$}

Once again ignoring absorption, we position the foci at the center of the crystal and consider the regime where $\beta$ is sufficiently large that $\exp \left(-4 s^{2}\right)(=\exp \{-4$ $\left.\left.\times\left[\left(\sigma^{\prime}-\sigma_{x}\right) / 4 \beta\right]^{2}\right\}\right)$ is essentially unity over the range of significant $|H|^{2}$. This means that within the crystal's acceptance angle the beam has uniform amplitude; equivalently, the length for coherent addition $l_{a}$ should be less than the crystal length $l$. The condition that $l_{a} \ll b_{y}$ again applies, and we assume strong focusing ( $b_{x}, b_{y} \ll l$ ) but continue to make the paraxial approximation that has been assumed throughout this treatment: modifications for the case of a large walk-off angle have been considered by Eckardt et al. ${ }^{30} h_{m}$ can now be written as

$$
\begin{aligned}
h_{m}= & \frac{2 \pi^{3 / 2} \mathbf{e}}{\xi_{x}} \int_{-\infty}^{\infty} \exp \left[-4\left(\frac{\sigma^{\prime}-\sigma}{4 \beta}\right)^{2}\right] \\
& \times\left|\frac{1}{2 \pi} \int_{-\infty}^{\infty} \exp \left[i \sigma^{\prime} \tau_{x}^{\prime} f\left(\tau_{x}^{\prime}\right) \mathrm{d} \tau_{x}{ }^{\prime}\right]\right|^{2} \frac{\mathrm{d} \sigma^{\prime}}{4 \beta},
\end{aligned}
$$

with

$$
f\left(\tau_{x}{ }^{\prime}\right)=\frac{1}{\left(1+i \tau_{x}{ }^{\prime}\right)^{1 / 2}\left(1+i \mathbf{e}^{2} \tau_{x}{ }^{\prime}\right)^{1 / 2}} g\left(\tau_{x}{ }^{\prime}\right),
$$

where the aperture function $g\left(\tau_{x}{ }^{\prime}\right)=1$ for $\left|\tau_{x}{ }^{\prime}\right|<\xi_{x}$ and $g\left(\tau_{x}{ }^{\prime}\right)=0$ elsewhere. We now invoke Parseval's theorem and find that

$$
\begin{aligned}
h_{m} & =\frac{\pi^{1 / 2} \mathbf{e}}{4 \beta \xi_{x}} \int_{-\infty}^{\infty}\left|f\left(\tau_{x}{ }^{\prime}\right)\right|^{2} \mathrm{~d} \tau_{x}{ }^{\prime} \\
& =\frac{\pi^{1 / 2} \mathbf{e}}{4 \beta \xi_{x}} \int_{-\xi_{x}}^{\xi_{x}}\left|\frac{1}{\left(1+i \tau_{x}{ }^{\prime}\right)^{1 / 2}\left(1+i \mathbf{e}^{2} \tau_{x}{ }^{\prime}\right)^{1 / 2}}\right|^{2} \mathrm{~d} \tau_{x}{ }^{\prime}
\end{aligned}
$$

The function of $\tau$ can be rewritten as

$$
\begin{aligned}
& \left|\left[\frac{1}{\left(1+i \tau_{x}{ }^{\prime}\right)\left(1+i \mathbf{e}^{2} \tau_{x}{ }^{\prime}\right)}\right]^{1 / 2}\right|^{2} \\
& =\frac{1}{\left(1+\tau_{x}{ }^{\prime 2}\right)^{1 / 2}\left(1+\mathbf{e}^{4}{\tau_{x}}^{\prime 2}\right)^{1 / 2}},
\end{aligned}
$$

and thus

$$
\begin{aligned}
h_{m} & =\frac{\pi^{1 / 2} \mathbf{e}}{4 \beta \xi_{x}} \int_{-\xi_{x}}^{\xi_{x}}\left[\left(1+\tau_{x}{ }^{2}\right)\left(1+\mathbf{e}^{4} \tau_{x}{ }^{2}\right)\right]^{-1 / 2} \mathrm{~d} \tau_{x}{ }^{\prime} \\
& =\frac{\pi^{1 / 2}}{2 \beta \xi_{x} \mathbf{e}} \int_{0}^{\xi_{x}}\left[\left(1+\tau_{x}{ }^{2}\right)\left(\mathbf{e}^{-4}+\tau_{x}{ }^{\prime 2}\right)\right]^{-1 / 2} \mathrm{~d} \tau_{x}{ }^{\prime}
\end{aligned}
$$

Equation (19) is now an incomplete elliptic integral of the first kind $^{31}$ :

$$
F(\phi \backslash \alpha)=a \int_{0}^{x}\left[\left(t^{2}+a^{2}\right)\left(t^{2}+b^{2}\right)\right]^{-1 / 2} \mathrm{~d} t,
$$

where $\tan \phi=x / b, \cos \alpha=b / a$, and $a>b$. In this case $a=1$ and $b=1 / \mathbf{e}^{2}(\mathbf{e}>1)$. The integral does not depend on $B$ and increases monotonically with ellipticity toward the state in which $h_{m}$ is optimized with respect to $\xi_{y}$ and independent of $\xi_{x}$. We therefore write $h_{m}$ in terms of $\xi_{y}$ :

$$
h_{m}=\frac{\pi^{1 / 2}}{2 B \xi_{y}{ }^{1 / 2}} F\left(\tan ^{-1} \xi_{y} \backslash \cos ^{-1} \frac{1}{\mathbf{e}^{2}}\right) .
$$

The optimal value of $\xi_{y}$ in the limit of high $B$ and $\mathbf{e}$ is thus found to be 3.3189 , to be compared with the limiting value of 1.3919 for $\mathbf{e}=1$ and with the optimal value of 2.84 for the complete version of $h_{m}$ when $B=0$. We note that Eq. (21) is inconsistent with the expression quoted by Adams and Ferguson, ${ }^{22}$ which depends equally on $w_{x}$ and $w_{y}$.

From our analytic function, with $B=16, \mathbf{e}=1$, and $\xi_{x}=\xi_{y}=1$, we obtain $h_{m}=\pi^{3 / 2} / 128=0.0435$. This result is in good agreement with the computed result of 0.0430 , which is lower by $1 \%$. In general, the approximation $\exp \left(-4 s^{2}\right)=1$ will make the analytic value an overestimate. 


\section{APPLICATION}

To determine the best focusing parameters for a specific crystal and wavelength, it is first necessary to calculate the phase-matching and walk-off angles from refractiveindex data such as Sellmeier coefficients. From the phase-matching angle and the refractive index can be calculated the crystal cut, for either normal or Brewsterangle incidence, and from the walk-off angle it is possible to work out the $B$ coefficient. The conversion efficiency and focusing parameters are then given by reference to Figs. 1 and 2.

For harmonic generation from beams of circular cross section, elliptical focusing will rarely be worth the losses and alignment difficulties introduced by the additional lenses. If a circular Gaussian beam is focused into the crystal at Brewster's angle, however, the beam within the crystal will be elliptical, with $\mathbf{e}=\eta$. For negative uniaxial crystals the ellipticity will unfortunately lie in the wrong direction if the crystal is cut to minimize reflection of the fundamental, as when it is used within an enhancement cavity. With positive uniaxial crystals, however, the ellipticity introduced by the Brewster-angled face will be in the correct sense.

Ring enhancement cavities usually include off-axis spherical mirrors, and the modes that they support thus have elliptical foci. The appropriate orientation of the doubling crystal will in such cases often not only tolerate this ellipticity but allow increased efficiency to follow from it; the same is true for the frequency doubling of diode lasers. For frequency quadrupling, ${ }^{6,7}$ the efficiency depends quadratically on that of the first doubling stage, and elliptical focusing could thus improve the overall conversion efficiency by a factor of 2 . In all cases care must be taken to ensure that the focal ellipticity and polarization are appropriately oriented.

The analysis presented in this paper is valid for all but the broadest-bandwidth lasers. Longitudinal coherence is required explicitly over a length $l_{1}=(\Delta k / k) l$, which corresponds to the longitudinal range that contributes to the instantaneous output intensity; this length is the result of the Guoy shift and will thus be of the order of a wavelength. It is also necessary, however, that the phase-matching condition be satisfied for the instantaneous wavelength, and thus dispersion of the birefringence should introduce a phase mismatch

$$
l \Delta k \sim l \frac{\mathrm{d}(\Delta k)}{\mathrm{d} \phi} \frac{\mathrm{d} \phi}{\mathrm{d} \lambda} \Delta \lambda \ll 1 .
$$

Hence [refer to Eq. (4)], the laser bandwidth is limited to $\Delta \lambda \sim 1 /\left(l k_{2} \rho \mathrm{d} \phi / \mathrm{d} \lambda\right)$, where $\mathrm{d} \phi / \mathrm{d} \lambda$ is the sensitivity of tuning angle to wavelength. For a typical example of doubling green light in a 7 -mm crystal of $\beta$-barium borate, this sensitivity is $\sim 0.1 \mathrm{~nm}$, making our results valid for most lasers with pulses longer than a few picoseconds.

\section{ACKNOWLEDGMENTS}

The authors thank the UK Science and Engineering Research Council and the Max-Planck-Gesellschaft for support during the course of this research. ${ }^{32}$
*Present address, Physical and Theoretical Chemistry Laboratory, Oxford University, South Parks Road, Oxford OX1 3QZ, UK. Phone, (+44)-1865-275421; fax, (+44)1865-275410; e-mail, timf@physchem.ox.ac.uk.

${ }^{\dagger}$ Present address, Morgan Grenfell Quantitative Investments, Ltd., 20 Finsbury Circus, London EC2M 1NB, UK.

${ }^{\text {}}$ Present address, Ion Storage Group, National Institute of Standards and Technology, Boulder, Colorado 80303-3328.

\section{REFERENCES}

1. G. D. Boyd and D. A. Kleinman, "Parametric interaction of focused Gaussian light beams," J. Appl. Phys. 39, 35973639 (1968).

2. J.-J. Zondy, "Comparative theory of walkoff-limited type-II versus type-I second harmonic generation with gaussian beams," Opt. Commun. 81, 427-440 (1991). "Note that in Eq. (3.1a) of that paper $\exp \left(-x^{2}\right)$ should read $\exp (-x / 2)$ "; see J. J. Zondy, M. Abed, and A. Clairon, "Type-II frequency doubling at $\lambda=1.30 \mu \mathrm{m}$ and $\lambda=2.53 \mu \mathrm{m}$ in flux-grown potassium titanyl phosphate," J. Opt. Soc. Am. B 11, 20042015 (1994).

3. S. C. Mehendale and P. K. Gupta, "Effect of double refraction on type II phase matched second harmonic generation," Opt. Commun. 68, 301-304 (1988).

4. See, for example, M. de Angelis, G. M. Tino, P. De Natale, C. Fort, G. Modugno, M. Prevedelli, and C. Zimmermann, "Tunable frequency-controlled laser source in the near ultraviolet based on doubling of a semiconductor diode laser," Appl. Phys. B 62, 333-338 (1996).

5. L. Goldberg and D. A. V. Kliner, "Tunable UV generation at $286 \mathrm{~nm}$ by frequency tripling of a high-power mode-locked semiconductor laser," Opt. Lett. 20, 1640-1642 (1995).

6. L. Goldberg and D. A. V. Kliner, "Deep-UV generation by frequency quadrupling of a high-power GaAlAs semiconductor laser," Opt. Lett. 20, 1145-1147 (1995).

7. C. Zimmermann, V. Vuletic, A. Hemmerich, and T. W. Hänsch, "All solid state laser source for tunable blue and ultraviolet radiation,” Appl. Phys. Lett. 66, 2318-2320 (1995).

8. Y. Taira, "High-power continuous-wave ultraviolet generation by frequency doubling of an argon laser," Jpn. J. Appl. Phys. 31, L682-L684 (1992).

9. V. D. Volosov and E. V. Nilov, "Effect of the spatial structure of a laser beam on the generation of the second harmonic in ADP and KDP crystals," Opt. Spectrosc. (USSR) 21, 392-394 (1966).

10. V. D. Volosov, "Effect of laser and medium parameters on second-harmonic generation," Sov. Phys. Tech. Phys. 14, 1652-1658 (1970).

11. P. Chmela, "Theory of second harmonic generation in focused light beam of fundamental frequency with the Gaussian distribution of light intensity under the interaction of type $o+o \rightarrow e$," Czech. J. Phys. B 24, 1-23 (1974).

12. F. M. Librecht and J. A. Simons, "Second harmonic generation in birefringent crystals by a Gaussian beam with an elliptical cross section," IEEE J. Quantum Electron. QE-11, 850-852 (1975).

13. D. J. Kuizenga, "Optimum focusing conditions for parametric gain in crystals with double refraction," Appl. Phys. Lett. 21, 570-572 (1972).

14. R. Fischer, P. V. Nickles, T. B. Chu, and L.-W. Wieczorek, "Optimum conditions for elliptical focusing in singly resonant optical parametric oscillators with hemispherical resonator," Ann. Phys. 39, 287-294 (1982).

15. T.-B. Chu and M. Broyer, "Intracavity cw difference frequency generation by mixing three photons and using Gaussian laser beams," J. Phys. (Paris) 46, 523-533 (1985).

16. L. P. Schelonka, M. Hofmann, and H. H. Klingenberg, "Optimum cylindrical focusing for laser frequency doubling," in 
Digest of the Fachsitzungen der Deutschen Physikalischen Gemeinschaft (Deutsche Physikalische Gesellschaft, Bad Honnef, Germany, 1992), p. 1445.

17. D. W. Coutts and J. A. Piper, "One watt average power by second harmonic and sum frequency generation from asingle medium scale copper vapor laser," IEEE J. Quantum Electron. 28, 1761-1764 (1992).

18. T. Omatsu, K. Kuroda, T. Takase, A. Hasegawa, and I. Ogura, "Second harmonic generation of a copper vapor laser using an anamorphic optical system," Jpn. J. Appl. Phys. 33 Pt 1, 4903-4904 (1994).

19. V. V. Rostovtseva and A. P. Sukhorukov, "Influence of thermal self-interaction on second harmonic generation in beams with an elliptic cross section," Sov. J. Quantum Electron. 13, 804-806 (1983).

20. W. Wang and M. Ohtsu, "Frequency-tunable sum- and difference-frequency generation by using two diode lasers in a KTP crystal," Opt. Commun. 102, 304-308 (1993).

21. N. Beverini, G. L. Genovesi, E. Maccioni, A. M. Messina, and F. Strumia, "Near-UV sub-Doppler spectroscopy on a metastable $\mathrm{Mg}$ beam by a frequency-doubled diode laser," Appl. Phys. B 59, 321-326 (1994).

22. C. S. Adams and A. I. Ferguson, "Frequency doubling of a single frequency Ti: $\mathrm{Al}_{2} \mathrm{O}_{3}$ laser using an external enhancement cavity," Opt. Commun. 79, 219-223 (1990).

23. S. Bourzeix, M. D. Plimmer, F. Nez, L. Julien, and F. Biraben, "Efficient frequency doubling of a continuous wave titanium:sapphire laser in an external enhancement cavity," Opt. Commun. 99, 89-94 (1993).

24. A. Steinbach, M. Rauner, F. C. Cruz, and J. C. Bergquist, " $\mathrm{Cw}$ second harmonic generation with elliptical Gaussian beams," Opt. Commun. 123, 207-214 (1996). It should be noted that there is a typographical error in Eq. (3) and that the curves for $\xi_{y}=3.3$ from Figs. 2 and 3 should be used instead of that for $B=16$ in Fig. 1, which is incorrect.

25. A. E. Siegman, Lasers (University Science, Mill Valley, Calif., 1986).

26. M. V. Hobden, "Phase-matched second-harmonic generation in biaxial crystals," J. Appl. Phys. 38, 4365-4372 (1967).

27. M. A. Dreger and J. H. Erkkila, "Improved method for calculating phase-matching criteria in biaxial nonlinear materials," Opt. Lett. 17, 787-788 (1992).

28. W. H. Press, B. P. Flannery, S. A. Teukolsky, and W. T. Vetterling, Numerical Recipes (Cambridge U. Press, Cambridge, 1986)

29. I. J. Evans, "High repetition rate tunable lasers," D. Phil. dissertation (Oxford University, Oxford, 1993).

30. R. C. Eckardt, H. Masuda, Y. X. Fan, and R. L. Byer, "Absolute and relative nonlinear optical coefficients of KDP, $\mathrm{KD}^{*} \mathrm{P}, \mathrm{BaB}_{2} \mathrm{O}_{4}, \mathrm{LiIO}_{3}, \mathrm{MgO}: \mathrm{LiNbO}_{3}$, and KTP measured by phase-matched second-harmonic generation," IEEE J. Quantum Electron. 26, 922-933 (1990).

31. M. Abramowitz and I. A. Stegun, Handbook of Mathematical Functions (National Bureau of Standards, Washington, D.C., 1964).

32. Preliminary findings were reported in T. G. M. Freegarde, J. Coutts, and A. Corney, "Second harmonic generation in beta-barium borate using elliptical focussing," in Nonlinear Optical Properties, Vol. 9 of 1988 OSA Technical Digest Series (Optical Society of America, Washington, D.C. 1988); paper MF5. The treatment of astigmatic Gaussian beams and improved numerical calculations are described by $\mathrm{D}$. Leibfried, "Präzisionsspektroskopie an atomarem Wasserstoff," Ph.D. dissertation (Max-Planck-Institut für Quantenoptik, Garching, Germany, 1995). 